\title{
Worldwide, 3-Year, Post-Marketing Surveillance Experience with Tofacitinib in Rheumatoid Arthritis
}

Stanley Cohen · Jeffrey R. Curtis - Ryan DeMasi · Yan Chen •

Haiyun Fan · Arif Soonasra · Roy Fleischmann

Received: November 13, 2017 / Published online: February 22, 2018

(C) The Author(s) 2018. This article is an open access publication

\begin{abstract}
Introduction: Post-marketing surveillance (PMS) is an integral part of monitoring adverse events (AEs) following approval of new drugs. Tofacitinib is an oral Janus kinase inhibitor for the treatment of rheumatoid arthritis (RA). An analysis of PMS reports was conducted to evaluate the safety of tofacitinib in a post-marketing setting.
\end{abstract}

Methods: Worldwide tofacitinib PMS data received in the Pfizer safety database from November 6, 2012 (first marketing authorization of tofacitinib) to November 5, 2015 were analyzed. Serious AEs (SAEs) of interest were

Enhanced content To view enhanced content for this article go to https://doi.org/10.6084/m9.figshare. 5853999.

S. Cohen · R. Fleischmann

Metroplex Clinical Research Center, Dallas, TX, USA

J. R. Curtis

University of Alabama at Birmingham, Birmingham, AL, USA

R. DeMasi

Pfizer Inc, New York, NY, USA

Y. Chen · H. Fan · A. Soonasra $(\varangle)$

Pfizer Inc, Collegeville, PA, USA

e-mail: arif.soonasra@pfizer.com

R. Fleischmann

University of Texas Southwestern Medical Center,

Dallas, TX, USA reviewed and reporting rates (RRs) were calculated by dividing the number of SAEs by the estimated 100 patient-years of exposure. Patient exposure was calculated based on estimated worldwide sales and an estimated daily regimen of tofacitinib $5 \mathrm{mg}$ twice daily.

Results: During the 3-year reporting period, worldwide post-marketing exposure to tofacitinib since approval was estimated to be 34,223 patient-years. In total, 9291 case reports $(82.9 \%$ non-serious) were received and 25,417 AEs, 102 fatal cases, and 4352 SAEs were reported. The RRs (per 100 patient-years) for SAEs of interest by Medical Dictionary for Regulatory Activities System Organ Class were 2.57 for infections, 0.91 for gastrointestinal disorders, 0.60 for respiratory disorders, 0.45 for neoplasms, 0.43 for cardiac disorders, and 0.12 for hepatobiliary disorders.

Conclusions: Although there are limitations to these data, no new safety risks were revealed in this real-world setting compared with the safety profile identified in the tofacitinib RA clinical development program. Any risks identified through the tofacitinib development program and PMS will continue to be monitored through pharmacovigilance surveillance.

Funding: Pfizer Inc.

Keywords: Disease-modifying antirheumatic drug; Post-marketing surveillance; Registry; Rheumatoid arthritis; Tofacitinib 


\section{INTRODUCTION}

Rheumatoid arthritis (RA) is a systemic, chronic autoimmune disease associated with joint pain and inflammation, potentially resulting in joint destruction. Tofacitinib is an oral Janus kinase inhibitor for the treatment of RA. Clinical efficacy and safety of tofacitinib have been established in numerous patient populations with active RA in phase 2 [1] and phase 3 [2-7] randomized controlled trials (RCTs), and long-term extension (LTE) studies $[8,9]$.

Tofacitinib received first marketing authorization in the United States (US) on November 6,2012 for the treatment of adults with moderately to severely active RA and an inadequate response/intolerance to methotrexate. As of November 2015 (data-cut for analysis), tofacitinib was approved in 46 countries and marketed in 29 countries. Tofacitinib has since received marketing authorization in the European Union and is currently approved in over 80 countries worldwide. In most countries, the approved tofacitinib dose for RA is $5 \mathrm{mg}$ twice daily (BID); a $10 \mathrm{mg}$ BID dose is approved in Botswana, Switzerland, and Russia. An extended-release formulation, dosed at $11 \mathrm{mg}$ once daily, has been approved in the US for the same indication.

Post-marketing surveillance (PMS) is integral to adverse event (AE) monitoring following regulatory approval of drugs. PMS complements phase 3 and LTE safety data to establish a realworld safety profile. A pooled analysis of tofacitinib-treated patients during phase 1, 2, 3, and LTE studies (to March 2015) found that AEs were generally stable over time, with no new safety risks versus previous reports [10]. PMS includes spontaneous or voluntary reporting of AEs, post-marketing observational studies, or active surveillance.

We aimed to evaluate the safety of tofacitinib in the post-marketing setting using spontaneous/non-interventional solicited PMS reports (i.e., market research where the product was not offered but AE data were collected) and non-study literature cases (i.e., cases not reported in a study but published as a case report) during the 3 years following first marketing authorization.

\section{METHODS}

\section{Data Sources}

This analysis reviewed worldwide tofacitinib PMS data in the Pfizer safety database from November 6, 2012 to November 5, 2015. Most were from post-marketing reports spontaneously submitted to Pfizer by healthcare professionals or patients. Additional data included non-interventional solicited reports (e.g., Pfizersponsored adherence or consumer support programs) and non-study literature case reports identified during routine pharmacovigilance review. The PMS data used in this analysis were not collected as part of a clinical study and were non-interventional, therefore ethics approval was not required.

\section{Estimated Worldwide Post-Marketing Exposure}

Worldwide exposure estimates are based on audited standard unit tofacitinib sales (SUs) from IMS Health's Multinational Integrated Data Analysis System (MIDAS) database through June 30, 2015. These were estimated by extrapolating the growth rate from the previous quarter, which was prorated to the end of the reporting period and converted into patientdays (days of therapy) using average daily dose. Patient-years were calculated by dividing patient-days by 365.25 .

The following are important considerations when utilizing data from the IMS Health databases. Tofacitinib is often sold in specialty pharmacies, which may not be captured in the MIDAS audit for all countries, potentially leading to under-reporting of units sold. Unit data from the MIDAS audit reflects units sold but not whether the drug was prescribed/taken by a patient. Furthermore, the IMS Prescriber Insights medical and prescription data used are not captured from all markets where tofacitinib is available. Therefore, total prescriptions are 
estimated by extrapolating from those where tofacitinib sales are captured. Additionally, the patient-years metric does not represent unique patient counts.

\section{Data Analysis}

For each case report, the type and frequency of serious AEs (SAEs) of interest by the Medical Dictionary for Regulatory Activities (MedDRA) System Organ Class (SOC) were reviewed; SAEs included infections, neoplasms, and gastrointestinal (GI), hepatobiliary, respiratory, and cardiac disorders. The seriousness of AEs was assessed using International Conference on Harmonisation criteria: an event was considered serious if it resulted in hospitalization or caused disability. The reporting rate (RR) for SAEs of special interest per MedDRA SOC by 6-month interval was estimated by dividing the SAE number by the estimated 100 patient-years of exposure. Multiple events could be reported in the same case report and were considered separate events. Percentages of AEs were calculated by dividing the case number [by MedDRA Preferred Term (PT) or SOC] by the total number of cases. Results are reported descriptively.

\section{RESULTS}

During the reporting period, the worldwide post-marketing exposure to tofacitinib, based on estimated worldwide sales and a regimen of tofacitinib $5 \mathrm{mg}$ BID, was 34,223 patient-years. Overall, 9291 case reports were received, including $56.3 \%$ reported by healthcare professionals; 9206 (99.1\%) were spontaneous reports, $77(0.8 \%)$ were from non-interventional solicited sources, and eight $(0.1 \%)$ were non-study literature cases. Most originated in the US (73.9\%). Other countries with large contributions (>100) included Canada, Argentina, Colombia, Australia, and Switzerland. Of cases with reported gender $(92.9 \%)$ or age $(79.2 \%)$, $78.2 \%$ occurred in females, and median age was 60 years. Of the cases where information on medical history was available, in addition to RA, the other most commonly reported ( $>150$ cases per PT) pre-existing medical conditions by PT included drug hypersensitivity, hypertension, drug ineffective, osteoarthritis, gastroesophageal reflux disease, diabetes mellitus, hypersensitivity, fibromyalgia, and hypothyroidism.

Overall, 25,417 AEs were reported through November 5, 2015; 82.9\% were non-serious. The MedDRA SOCs with the most reported AEs included general, GI, and musculoskeletal disorders. The most reported AEs by PT (Fig. 1) included drug ineffective (13.2\%), headache $(9.0 \%)$, pain $(6.4 \%)$, fatigue $(6.0 \%)$, nausea $(6.0 \%)$, condition aggravated $(5.9 \%)$, diarrhea (5.8\%), and arthralgia (5.2\%).

Overall, 4352 SAEs were reported; RRs for SAEs of special interest by SOC are presented in Table 1 . The most reported SAEs by PT (Fig. 2) included RA (4.5\%), condition aggravated (3.4\%; any condition [RA or other] that worsened), pneumonia (1.7\%), and arthropathy $(1.2 \%)$.

\section{Infections}

Serious infections were the most reported SAEs by SOC (879 events), with an RR of 2.57 per 100 patient-years. Estimated RR was highest in the first year and generally decreased over time. The most common serious infections by PT (number of events) were pneumonia (158), lower respiratory tract/respiratory tract infection (71), urinary tract infection (53), diverticulitis (37), kidney infection (35), sepsis (31), Staphylococcal infection (28), cellulitis (25), lung infection (24), and infection (21).

Several SAEs with PTs (events) indicative of potential opportunistic infections (OIs) were identified, including disseminated herpes zoster (HZ; seven), tuberculosis (six), pneumonia cryptococcal (three), Candida infection/esophageal candidiasis/oropharyngeal candidiasis (two each), histoplasmosis (two), cytomegalovirus colitis/gastritis/esophagitis (one each), Pneumocystis jirovecii pneumonia (one), and unspecified OI (two). Two histoplasmosis events (not previously reported in tofacitinib RCTs) were reported. 


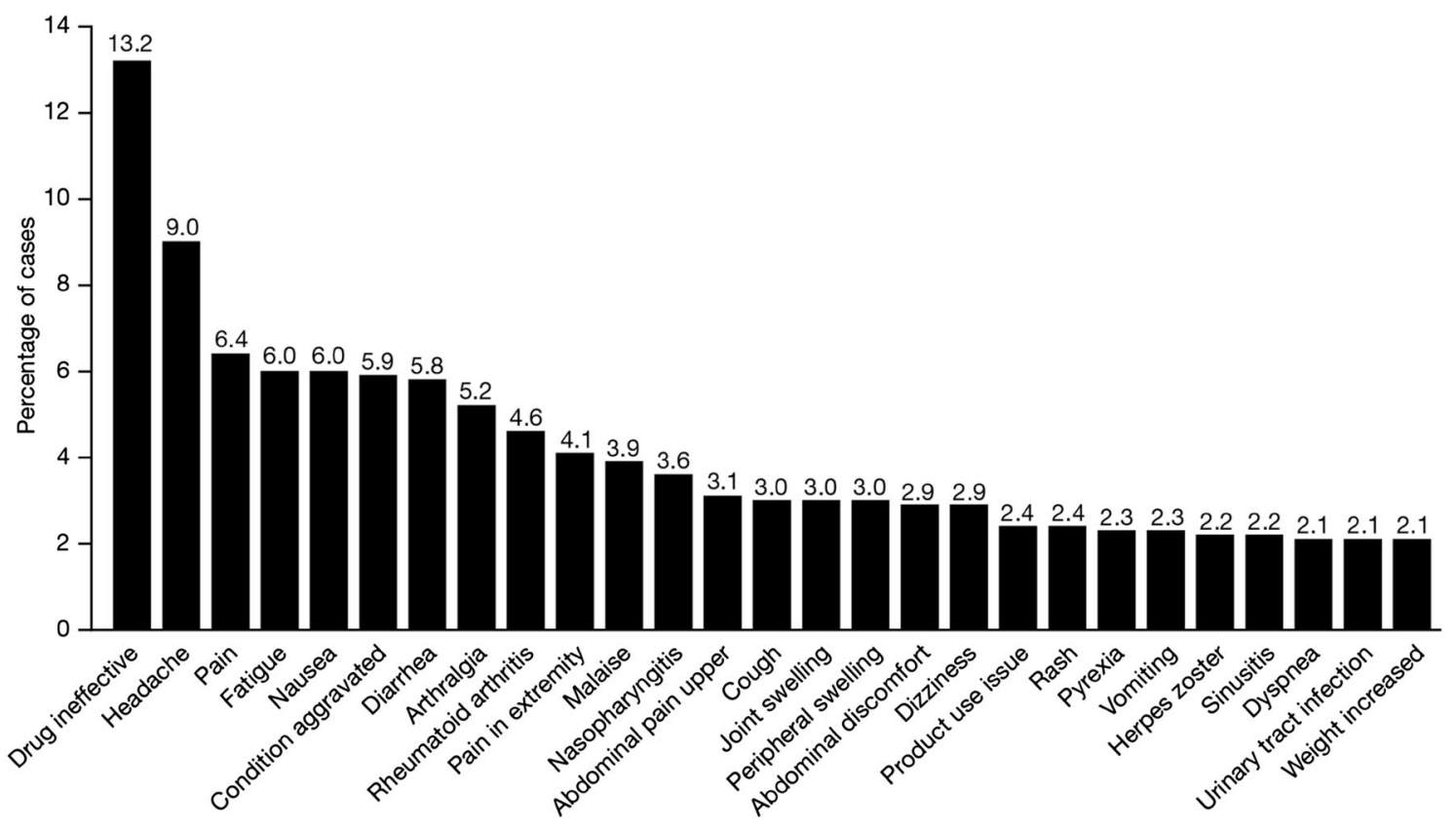

Fig. 1 Most frequently reported AEs based on MedDRA PTs (reporting proportion $\geq 2 \%$ ). The percentage was calculated by dividing the number of cases per PT by the total number of cases. The sum of the percentages may be

\section{Neoplasms}

The most reported neoplasm SAEs by PT (events) included lymphoma (13), neoplasm malignant (11), skin cancer (ten), lung neoplasm malignant (eight), basal cell carcinoma (eight), breast cancer (six), squamous cell carcinoma (six), malignant melanoma (six), brain neoplasm (five), squamous cell carcinoma of the skin (four), prostate cancer (four), uterine cancer (four), and colon cancer (four).

Non-melanoma skin cancer (NMSC) was the most reported malignancy in RA patients receiving tofacitinib in PMS, with 16 serious (as assessed by case processor) cases of NMSC reported, including basal cell carcinoma (eight) and squamous cell carcinoma (ten; two cases had both basal and squamous cell carcinoma). Ten cases with unspecified skin cancer were reported.

There were 15 reported cases of lymphoma by PT (events), including lymphoma (13), B-cell lymphoma (two), and extranodal marginal zone B-cell lymphoma (one). One case was reported more than $100 \%$ due to patients with multiple AEs. $A E$ adverse event, $M e d D R A$ Medical Dictionary for Regulatory Activities, $P T$ preferred term

with systemic diffuse large B-cell lymphoma with central nervous system involvement. Three others were reported with lymphoma and unspecified brain neoplasm, however it was unclear whether the brain neoplasm events were secondary to the spread of lymphoma. There was no increase in reporting frequency of lymphoma over time.

Estimated RRs for neoplasm events by 6-month interval ranged from 0.14 to 0.69 per 100 patient-years, with an overall rate of 0.45 per 100 patient-years (Table 1). The RR was highest after the first year and stabilized thereafter.

\section{GI and Hepatobiliary Events}

The most reported GI-related SAEs (events) included diarrhea (24), nausea (24), abdominal pain/abdominal pain upper (21), vomiting (20), hematochezia (15), GI perforation (nine), GI hemorrhage/GI disorder (eight each), and pancreatitis (eight). RRs appeared to decrease over time, with an RR of 0.91 per 100 patient-years. 
Table 1 Exposure and reporting rate of deaths and SAEs by 6-month reporting period (November 2012-November 2015)

\begin{tabular}{|c|c|c|c|c|c|c|c|}
\hline $\begin{array}{l}\text { Reporting } \\
\text { interval }\end{array}$ & $\begin{array}{l}\text { Interval } 1 \\
06 \text { Nov } \\
2012-05 \\
\text { May } 2013\end{array}$ & $\begin{array}{l}\text { Interval } 2 \\
06 \text { May } \\
2013-05 \\
\text { Nov } 2013\end{array}$ & $\begin{array}{l}\text { Interval } 3 \\
06 \text { Nov } \\
2013-05 \\
\text { May } 2014\end{array}$ & $\begin{array}{l}\text { Interval } 4 \\
06 \text { May } \\
2014-05 \\
\text { Nov } 2014\end{array}$ & $\begin{array}{l}\text { Interval } 5 \\
06 \text { Nov } \\
2014-05 \\
\text { May } 2015\end{array}$ & $\begin{array}{l}\text { Interval } 6 \\
06 \text { May } \\
2015-05 \\
\text { Nov } 2015\end{array}$ & Overall \\
\hline $\begin{array}{l}\text { Patient-years of } \\
\text { exposure }^{\mathrm{a}}\end{array}$ & 709 & 2623 & 4511 & 6719 & 8888 & 10,733 & 34,223 \\
\hline $\begin{array}{l}\text { Number of cases } \\
\text { reported }\end{array}$ & 368 & 1000 & 1131 & 1575 & 2026 & 3196 & 9291 \\
\hline $\begin{array}{l}\text { Number of AEs } \\
(\mathrm{RR})^{\mathrm{b}}\end{array}$ & $957(135.0)$ & $\begin{array}{l}2720 \\
\quad(103.7)\end{array}$ & $2918(64.7)$ & $4221(62.8)$ & $6236(70.2)$ & $8365(77.6)$ & $25,417(74.3)$ \\
\hline $\begin{array}{l}\text { Number of } \\
\text { SAEs }(R R)^{b}\end{array}$ & $176(24.8)$ & $446(17.0)$ & $517(11.6)$ & $714(10.6)$ & $1137(12.8)$ & 1137 (12.8) & $4352(12.7)$ \\
\hline $\begin{array}{l}\text { Number of fatal } \\
\text { cases }(R R)^{b}\end{array}$ & $3(0.42)$ & $10(0.38)$ & $12(0.27)$ & $16(0.24)$ & $24(0.27)$ & $37(0.34)$ & $102(0.30)$ \\
\hline \multicolumn{8}{|c|}{ Number of SAEs and reporting rate per 100 patient-years by $S \mathrm{CC}^{\mathrm{b}}$} \\
\hline Infections & $38(5.36)$ & $103(3.93)$ & $100(2.22)$ & $148(2.20)$ & $217(2.44)$ & $273(2.53)$ & $879(2.57)$ \\
\hline GI disorders & $13(1.83)$ & $34(1.30)$ & $50(1.11)$ & $54(0.80)$ & $73(0.82)$ & $87(0.81)$ & $311(0.91)$ \\
\hline $\begin{array}{l}\text { Cardiac } \\
\text { disorders }\end{array}$ & $11(1.55)$ & $23(0.88)$ & $26(0.58)$ & $24(0.36)$ & $32(0.36)$ & $31(0.29)$ & $147(0.43)$ \\
\hline $\begin{array}{l}\text { Respiratory } \\
\text { disorders }\end{array}$ & $11(1.55)$ & $31(1.18)$ & $36(0.80)$ & $32(0.48)$ & $33(0.37)$ & $64(0.59)$ & $207(0.60)$ \\
\hline Neoplasms & $1(0.14)$ & $18(0.69)$ & $17(0.38)$ & $31(0.46)$ & $40(0.45)$ & $47(0.44)$ & $154(0.45)$ \\
\hline $\begin{array}{l}\text { Hepatobiliary } \\
\text { disorders }\end{array}$ & $1(0.14)$ & $3(0.11)$ & $2(0.04)$ & $9(0.13)$ & $13(0.15)$ & $13(0.12)$ & $41(0.12)$ \\
\hline
\end{tabular}

$A E$ adverse event, BID twice daily, GI gastrointestinal, MedDRA Medical Dictionary for Regulatory Activities, Nov November, $R R$ reporting rate per 100 patient-years, $S A E$ serious adverse event, $S O C$ system organ class

${ }^{a}$ Estimated based on marketing sales and an estimated daily regimen of tofacitinib $5 \mathrm{mg}$ BID

${ }^{b}$ Reporting rate (different from incidence rate) is calculated by dividing the number of SAEs per MedDRA SOC or deaths by the estimated patient-years of exposure (per 100 patient-years). Note, multiple events can be reported within the same case report

Diarrhea, nausea, vomiting, and abdominal pain represent identified adverse drug reactions (ADRs; AEs caused by the drug) of tofacitinib. Most patients with GI hemorrhage reports had pre-existing GI conditions, or were receiving concomitant non-steroidal anti-inflammatory drugs or anticoagulants. Of the eight cases of GI hemorrhage/GI disorder reported post-marketing, two involved the same patient who was hospitalized twice ( 1 month apart) due to the same event. The patient recovered from both episodes. Although a possible causal 


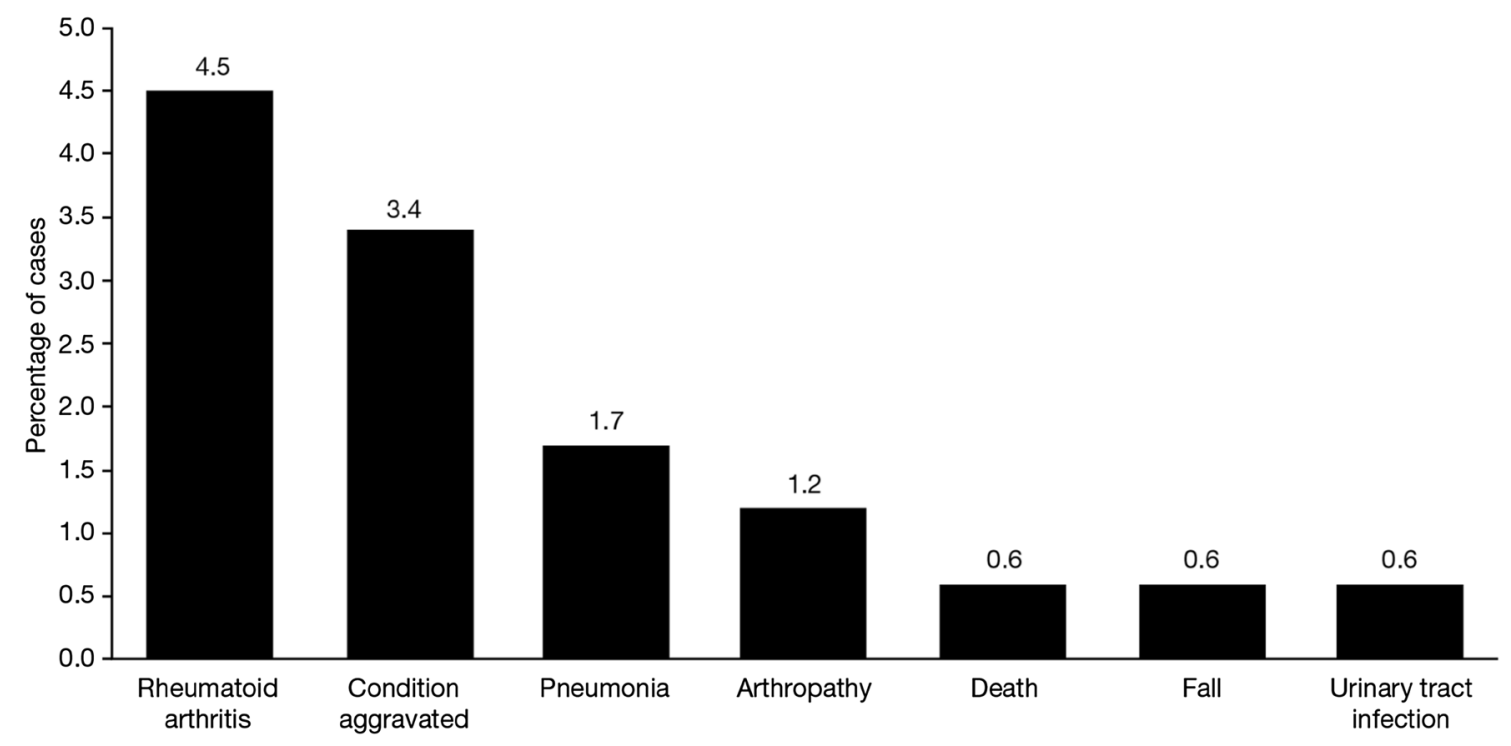

Fig. 2 Most frequently reported SAEs based on MedDRA PTs. The percentage was calculated by dividing the number of SAE cases per PT by the total number of cases. The sum of the percentages may be more than $100 \%$

relationship between the event and tofacitinib could not be excluded, concomitant methotrexate, prednisone, and naproxen may have contributed. Limited information was available for the remaining cases.

There were 25 cases reported with PTs (events) indicative of potential GI perforation, including GI perforation (nine), large intestinal perforation (four), intestinal perforation (three), diverticular perforation (three), appendicitis perforated (two), gastric perforation (two), gastric ulcer perforation (one), small intestinal hemorrhage (one), and GI hemorrhage (one). One patient recorded GI perforation and intestinal perforation. In two cases with reported intestinal hemorrhage, concomitant drugs (e.g., methotrexate, ibuprofen, prednisone, meloxicam) or medical history/concurrent conditions (e.g., diverticulitis, bowel infection) could not be excluded from contributing to these events, together with tofacitinib treatment.

\section{Respiratory Events}

Overall, 207 respiratory SAEs were reported (estimated RR: 0.60 per 100 patient-years). The due to patients with multiple AEs. $A E$ adverse event, MedDRA Medical Dictionary for Regulatory Activities, $P T$ preferred term, $S A E$ serious adverse event

most reported SAEs by PT (event) were dyspnea (44; tofacitinib ADR), pulmonary embolism (15), interstitial lung disease (ILD)/pulmonary fibrosis (15), chronic obstructive pulmonary disease (13), pulmonary edema (ten), pulmonary thrombosis (nine), epistaxis (eight), pulmonary mass (seven), cough (six), and pleural effusion (six).

ILD events are an important potential risk for tofacitinib. Of the 207 respiratory SAEs by PT, nine cases were ILD and six were pulmonary fibrosis; two reported previous history of ILD.

\section{Cardiac Events}

Overall, 147 cardiac events were recorded, with a numeric decrease in estimated RR over time, from 1.55 to 0.29 per 100 patient-years at the first 6-month interval and final 6-month interval, respectively. The most reported events by PT (events) included myocardial infarction (29), cardiac failure/cardiac failure congestive (21), cardiac disorder (19), atrial fibrillation (17), cardiac arrest (five), coronary artery disease (four), pericardial effusion (four), and tachycardia (three). 


\section{Deaths}

There were 102 deaths during the reporting period, giving an overall mortality rate of 0.3 per 100 patient-years, which was stable over time (Table 1). Of the 36 events with available cause of death information, reported causes (events) were infection-related AEs (15), cardiovascular events (eight), stroke (four), pulmonary embolism (two), ILD (two), demyelination (one), Stevens-Johnson syndrome (one), and procedure complications (one). Efforts to obtain additional information for the remaining cases are ongoing; cases will be re-assessed as information becomes available.

\section{DISCUSSION}

It is estimated that, as of August 2017, 102,214 patients worldwide have received tofacitinib (data on file); it is therefore important that tofacitinib safety is evaluated in patients with real-world experience. This analysis evaluated PMS reports for tofacitinib in RA over a 3-year period after first marketing authorization. AEs were consistent with the safety profile observed during RCTs $[1-8,10]$.

AEs were presented by PT, as opposed to SOC; however, some of the PTs relate to loss of efficacy rather than ADRs. For example, 'drug ineffective' was the most reported $\mathrm{AE}$. The most recorded SAEs by SOC also related to loss of efficacy of tofacitinib, followed by serious infections; however, the RR of these events decreased over time. A small number of potential OI SAEs included disseminated $\mathrm{HZ}$ and tuberculosis, consistent with the incidence of HZ [11], tuberculosis, and other OIs [12] observed across tofacitinib RA RCTs. Baseline tuberculosis screening status was not included in the PMS reports. Two histoplasmosis cases were recorded in the PMS; this was not reported during tofacitinib RA RCTs, but both cases originated in US regions where histoplasmosis is endemic.

Consistent with the tofacitinib label, the most common GI SAEs by PT were diarrhea, nausea, vomiting, and abdominal pain; all known ADRs for tofacitinib. Pancreatitis and GI perforations were reported in PMS and risk of these events is monitored though pharmacovigilance surveillance; however, potential contributory factors include concomitant medications (e.g., methotrexate and prednisone). Although pancreatitis has been reported in RA RCTs and PMS, data do not indicate an increased risk of pancreatitis with tofacitinib.

The types of malignancies reported in PMS were consistent with tofacitinib RA RCTs. The most common neoplasms reported in both settings were NMSCs. Overall neoplasm rates in the tofacitinib RA clinical program and PMS did not increase with increasing tofacitinib exposure [13]. In PMS, 15 lymphoma cases were reported over approximately 34,000 patientyears of exposure as of November 5,2015 . The RR of lymphoma did not increase over time. Risk of lymphoma with tofacitinib continues to be monitored through routine and enhanced pharmacovigilance activities. Consistent with data from tofacitinib RA LTEs, PMS data do not suggest an increased risk of cardiac events with tofacitinib.

Review of reported deaths did not suggest an increased risk of death with tofacitinib; no new safety information was identified versus RCTs. The most reported causes were consistent with those observed during tofacitinib RA RCTs, including infections and cardiovascular events.

The following limitations should be considered. Case reports are often submitted voluntarily; the magnitude of under-reporting is unknown. Importantly, some clinical information is often missing or incompletely recorded. Patients' age, pre-existing comorbidities, underlying RA, and concomitant medications could not be excluded as contributing to AEs, and due to the limited information on medical history captured in the spontaneous reports, it is not feasible to analyze the reported events by patients' pre-existing conditions to tease out any confounding effects. The spontaneous reporting system utilized in PMS yields RRs rather than incidence rates, limiting betweendrug comparisons.

Furthermore, RRs were calculated at the event level; there may have been correlations for events that occurred within the same patients. The RR may therefore be different at 
the patient level, and it cannot be adjusted for patient demographic characteristics. Patient exposure in PMS is calculated based on sales data rather than clinical trial data; comparisons between PMS and integrated safety database rates cannot be made. Additionally, it is difficult to make age- and gender-adjusted comparisons with the non-tofacitinib RA population. PMS data for other biologic therapies for RA have been published in patients with RA; however, in contrast to the worldwide tofacitinib data presented here, these reports only present countryspecific data [14, 15]. Additional disproportionality analysis (e.g., proportional reporting ratios) or Multi-Item Gamma Poisson Shrinkage would enable between-drug comparisons, although these are beyond the scope of this analysis. Furthermore, reported rates are based on estimated exposure based on commercial records, rather than actual exposure (i.e., exposure time was not censored at time of event). A known phenomenon in drug reporting is the Weber effect, which predicts higher AE RRs within the first 2 years of drug approval, decreasing thereafter, possibly due to reduced reporting of mild AEs. The data analyzed here were recorded within 3 years of marketing authorization; therefore, the Weber effect should be considered when interpreting early estimated RRs. Finally, an accumulation of AE reports may not necessarily indicate causation by the drug, and may relate to underlying diseases, comorbidities, or other factors. Despite these limitations, PMS data are important for characterizing safety profiles of medications in a real-world setting, including possible detection of new safety signals.

\section{CONCLUSIONS}

Based on this review of PMS data for tofacitinib in RA, the types of AEs and RRs were consistent with the known tofacitinib safety profile, with no new safety risks identified. Risks identified during the tofacitinib development program continue to be monitored through pharmacovigilance surveillance.

\section{ACKNOWLEDGEMENTS}

Funding. This study was funded by Pfizer Inc. Article processing charges were funded by Pfizer Inc.

Authorship. All named authors meet the International Committee of Medical Journal Editors (ICMJE) criteria for authorship for this article, take responsibility for the integrity of the work as a whole, and have given their approval for this version to be published. All authors had full access to all of the data in this study and take complete responsibility for the integrity of the data and accuracy of the data analysis.

Medical Writing and/or Editorial Assistance. Medical writing support, under the direction of the authors, was provided by Karleen Nicholson, $\mathrm{PhD}$, on behalf of CMC CONNECT, a division of Complete Medical Communications Ltd, Macclesfield, UK and Christina Viegelmann, PhD, of CMC CONNECT, a division of Complete Medical Communications Ltd, Glasgow, UK and funded by Pfizer Inc, New York, NY, USA in accordance with Good Publication Practice (GPP3) guidelines (Ann Intern Med 2015; 163:461-464).

Disclosures. Stanley Cohen has received consultancies, speaking fees, and honoraria from AbbVie, Amgen, Astellas, BMS, Lilly, Genentech, Gilead, Janssen, Novartis, Pfizer Inc, Roche, and Sandoz. Jeffrey R. Curtis has received consultancies, speaking fees, and honoraria from AbbVie, Amgen, BMS, Corrona, Janssen, Lilly, Myriad, Pfizer Inc, Roche/Genentech, and UCB; and has received research grants paid to the University of Alabama at Birmingham. Roy Fleischmann has received consultancies, speaking fees, and honoraria from AbbVie, Amgen, BMS, GSK, Janssen, Lilly, Novartis, Pfizer Inc, Sanofi-Aventis, and UCB. Ryan DeMasi is an employee and shareholder of Pfizer Inc. Yan Chen is an employee and shareholder of Pfizer Inc. Haiyun Fan is an employee and shareholder of Pfizer Inc. Arif Soonasra is an employee and shareholder of Pfizer Inc. 
Compliance with Ethics Guidelines. The PMS data used in this analysis were not collected as part of a clinical study and were noninterventional, therefore ethics approval was not required.

Data Availability. The datasets generated and/or analyzed during the current study are available from the corresponding author on reasonable request.

Open Access. This article is distributed under the terms of the Creative Commons Attribution-NonCommercial 4.0 International License (http://creativecommons.org/licenses/ by-nc/4.0/), which permits any noncommercial use, distribution, and reproduction in any medium, provided you give appropriate credit to the original author(s) and the source, provide a link to the Creative Commons license, and indicate if changes were made.

\section{REFERENCES}

1. Fleischmann R, Kremer J, Tanaka Y, et al. Efficacy and safety of tofacitinib in patients with active rheumatoid arthritis: review of key Phase 2 studies. Int J Rheum Dis. 2016;19:1216-25.

2. van der Heijde D, Tanaka Y, Fleischmann R, et al. Tofacitinib (CP-690,550) in patients with rheumatoid arthritis receiving methotrexate: twelve-month data from a twenty-four-month phase III randomized radiographic study. Arthritis Rheum. 2013;65:559-70.

3. Burmester GR, Blanco R, Charles-Schoeman C, et al. Tofacitinib (CP-690,550) in combination with methotrexate in patients with active rheumatoid arthritis with an inadequate response to tumour necrosis factor inhibitors: a randomised phase 3 trial. Lancet. 2013;381:451-60.

4. Fleischmann R, Kremer J, Cush J, et al. Placebocontrolled trial of tofacitinib monotherapy in rheumatoid arthritis. N Engl J Med. 2012;367:495-507.

5. Kremer J, Li ZG, Hall S, et al. Tofacitinib in combination with nonbiologic disease-modifying antirheumatic drugs in patients with active rheumatoid arthritis: a randomized trial. Ann Intern Med. 2013;159:253-61.

6. Lee EB, Fleischmann R, Hall S, et al. Tofacitinib versus methotrexate in rheumatoid arthritis. N Engl J Med. 2014;370:2377-86.

7. van Vollenhoven RF, Fleischmann R, Cohen S, et al. Tofacitinib or adalimumab versus placebo in rheumatoid arthritis. N Engl J Med. 2012;367:508-19.

8. Wollenhaupt J, Silverfield J, Lee EB, et al. Tofacitinib, an oral Janus kinase inhibitor, in the treatment of rheumatoid arthritis: safety and efficacy in open-label, long-term extension studies over 8 years. Arthritis Rheumatol. 2016;68(S10):2056-8.

9. Wollenhaupt J, Silverfield J, Lee EB, et al. Safety and efficacy of tofacitinib, an oral Janus kinase inhibitor, for the treatment of rheumatoid arthritis in open-label, long-term extension studies. J Rheumatol. 2014;41:837-52.

10. Cohen SB, Tanaka Y, Mariette X, et al. Long-term safety of tofacitinib for the treatment of rheumatoid arthritis up to 8.5 years: integrated analysis of data from the global clinical trials. Ann Rheum Dis. 2017;76:1253-62.

11. Winthrop KL, Yamanaka $H$, Valdez $H$, et al. Herpes zoster and tofacitinib therapy in patients with rheumatoid arthritis. Arthritis Rheumatol. 2014;66:2675-84.

12. Winthrop KL, Park SH, Gul A, et al. Tuberculosis and other opportunistic infections in tofacitinibtreated patients with rheumatoid arthritis. Ann Rheum Dis. 2016;75:1133-8.

13. Curtis JR, Lee EB, Kaplan IV, et al. Tofacitinib, an oral Janus kinase inhibitor: analysis of malignancies across the rheumatoid arthritis clinical development programme. Ann Rheum Dis. 2016;75:831-41.

14. Koike T, Harigai M, Inokuma S, et al. Postmarketing surveillance of tocilizumab for rheumatoid arthritis in Japan: interim analysis of 3881 patients. Ann Rheum Dis. 2011;70:2148-51.

15. Mendes D, Alves C, Batel Marques F. Safety of biologics approved for treating rheumatoid arthritis: analysis of spontaneous reports of adverse events. Clin Rheumatol. 2013;32:1207-14. 